Eur. Surg. Res. 1987;19:337-338

\title{
Prof. Dr. med. Dr. med. h.c. Walter Brendel - 65 Years
}

Scholars, colleagues and friends honor and celebrate Prof. Walter BrendeГs 65th birthday on November 6th, 1987 in Munich (FRG) with a scientific symposium, entitled 'Surgical Research: Accomplishments and Perspectives'.

Prof. Brendel was born in Karlsruhe on November 6th, 1922. His medical education at the University of Heidelberg was interrupted when he served in the German army during World War II in Russia, where he was severely injured. Following the war in 1948 he resumed his studies at the University of Heidelberg, receiving his medical degree (Dr. med.) in 1949. After rotating through internships in surgery, ophthalmology and internal medicine he entered the Department of Physiology under the direction of Prof. H. Schäfer and Prof. R. Thauer in the Max-Planck Society's Kerckhoff Institute for Cardiac Research in Bad Nauheim. In 1959 he became Privat-dozent for Physiology at the University of Giessen. During the years in Bad Nauheim and Giessen his research interests were focused mainly on cardiopul-monary physiology related to the effects of anesthesia on the cardiovascular system and lung function, temperature regulation, pathophysiology of deep hypothermia and extracorporeal circulation. At this early date in his work with extracorporeal circulation Dr. Brendel established scientific contacts with cardiovascular surgeons who were at that time employing this technique in open-heart surgery. In 1962, Prof. Rudolf Zenker, chairman of the Department of Surgery at the University of Munich proposed to Dr. Brendel to establish a laboratory for experimental surgery at the Surgical University Clinic in an effort to promote surgical research in his institution.

In accepting this challenge Walter Brendel quickly transformed his interests from general physiology to surgical pathophysiology with its multitude of fascinating aspects, recognizing early on that experimental

338

Prof. Dr. med. Dr. med. h.c. Walter Brendel - 65 Years 
surgery or surgical research can by no means be limited to improvement of surgical techniques and decisions alone, but, instead, must involve the creation of new surgical concepts backed by scientific exploration with emphasis on clinical application.

With his characteristic pragmatic approach to scientific problems Walter Brendel engaged himself and his research team in, among other things, transplantation research, which appeared to him at the time to be a more successful approach for coping with organ failure as opposed to the use of artificial organs. Together with his pupils and collaborators he made important contributions to immunosuppressive treatment in transplant recipients (antilymphocytic serum and ALG). From 1968 to 1982 Dr. Brendel headed a project entitled 'Restitution and substitution of internal organs' sponsored by the Deutsche Forschungsge-meinschaft. Within the framework of this program the basis for experimental and clinical transplantation immunology and organ transplantation was firmly established at the University of Munich which today has evolved into one of the most active and successful transplant centers in Germany. However, in addition to the many contributions to transplantation immunology Dr. Brende $\Gamma$ s Institute for Surgical Research in Munich enjoys international recognition in a wide range of research programs including surgical pathophysiology, microcirculation research, shock, blood replacement and hemodilution, brain edema, secondary brain injury, gastric physiology, wound healing, effects of anesthetics on the circulatory and immune systems, surgical infections, transplantation cytology, hypothermia as well as high altitude medicine. Since 1978, Dr. BrendeГs Institute for Surgical Research has been housed in an elaborately equipped new building complex located at the Klinikum Grosshadern of the University of Munich.

In 1975, with his gift for innovative scientific approach together with his untiring enthusiasm, Walter Brendel laid down the ground work for scientific exploration for the utilization of shock waves in clinical medicine. Upon its introduction into the clinical setting this new approach to a very old surgical problem (kidney stones) was met with widespread scepticism. Today, the use of shock waves in the treatment of kidney and biliary stones represents a unique example of the importance of surgical research, namely the introduction of new nonsurgical modalities for treatment of surgical diseases.

In 1967, Dr. Brendel became the first professor of experimental surgery in Munich, being honored in 1969 with the first chair of experimental surgery in Germany.

In 1968, he was elected president of the European Society for Experimental Surgery. As one of the founders of the journal European Surgical Research he was editor in chief during its first years and again from 1978 to present.

In his scientific career, Walter Brendel has inspired and educated a great number of researchers and scholars from all parts of the world; in doing so language barriers presented no hindrance for him, constantly breaking international constraints in order to convey his ideas and the enthusiasm necessary to pursue them. Thus, over the years, his institute has provided tuition for a great number of foreign research fellows from all medical disciplines. Walter Brende $\Gamma$ s significant contributions to the field of surgical research and clinical surgery in this country are reflected in the great number of important positions held by his disciples throughout Germany. Through his own scientific contribution as well as through the long list of scholars and colleagues who have received their basic research training under his guidance, Walter Brende $\Gamma$ s presence in the field of surgical research will be evident for generations to come.

Among scientists in the field of medical and surgical research Walter Brendel is one of the few who has implemented truly novel and multidisciplinary approaches to medical problems. His pupils and collaborators have received distinguished awards and recognitions from many nonsurgical international institutions. Walter Brendel himself received, in 1971, a doctor honoris causae from the University of São Paulo, Brasil; the German Society for Surgery acknowledged his extensive contributions to surgery by bestowing him with the Jubilee Award of the German Society for Surgery in 1977; his innovative contributions and ideas for the application of shock waves in medicine were honored in 1985 with the Körber Award for the Advancement of European Sciences.

On behalf of the members of the editorial board as well as the authors of this journal I take this opportunity to thank Walter Brendel for his endurance and devotion in making European Surgical Research a flourishing journal, with the very best of wishes on his 65 th birthday.

Prof. K. Messmer, Heidelberg, FRG 